\title{
VALID (RE-)USE OF MODELS-OF-THE-PHYSICS IN CYBER-PHYSICAL SYSTEMS USING VALIDITY FRAMES
}

\author{
Bert Van Acker \\ Paul De Meulenaere \\ Joachim Denil \\ University of Antwerp, BELGIUM \\ Flanders Make, BELGIUM \\ \{Bert.Vanacker,Paul.Demeulenaere,Joachim.Denil\}@uantwerpen.be
}

\author{
Yuri Durodie \\ Kris Vanstechelman \\ Alexander Van Bellinghen \\ Siemens PLM Software, BELGIUM \\ DANA BELGIUM NV, BELGIUM \\ kris.vanstechelman@dana.com \\ \{yuri.durodie,alexander.van_bellinghen\}@ siemens.com
}

\begin{abstract}
Modelling and Simulation techniques play a key role in the design of Software-Intensive and Cyber-Physical Systems. They enable not only early virtual integration of the different parts of the system but also the use of the dynamic behavior of the physical system in the control algorithms, to cope with the continuous demand for improved system control and performance. These physics-based high-fidelity models are mostly numerically-intensive that cannot be deployed on restricted embedded targets without altering the model. Altering these high-fidelity models, e.g. by performing mathematical reductions, decreases the model (re-)usability if the range-of-validity of this model is not formally captured. In this paper, we report on a framework to formally capture the range-of-validity of models-of-the-physics. The needed meta-information to grasp the range-of-validity is captured within a Validity Frame, together with the necessary experimenting and selection methods to increase re-use of physics-based system models. An academic case study is performed to demonstrate the proposed methodology.
\end{abstract}

Keywords: Validity Frame, Models-of-the-physics, Deployment, Model reuse.

\section{INTRODUCTION}

Development of software intensive systems, found for example in the avionics and automotive domains, are becoming increasingly more complex. One of the causes of this complexity is the synergistically interaction between software and physical elements (Lee 2008). Another cause is the demand for performance improvement (EMPHYSIS 2018) of these systems to enable e.g. faster and safer control, autonomous driving, reduced energy consumption and emission, etc. This can be achieved by using a model of the physics of the system to be controlled or monitored. These physics-based models enable predicting the behavior of the system in its valid operating region which can be utilized in advanced control or observers/virtual sensors, achieving significantly optimized control and/or system monitoring (Sánchez et al. 2017, Zeyu et al. 2017). 
High-fidelity models-of-the-physics are typically described as differential- and algebraic equations and are mostly numerically-intensive models. Running these numerically-intensive models on constrained embedded controllers with limited resources and computational performances can be very challenging or even impossible, especially with hard real-time constraints (Pastorino et al. 2016). Generally, the high-fidelity models need to be transformed to a simpler model which is suited for embedded controller deployment. For this transformation, numerous reduction techniques can be used such as order reductions, mathematical reductions, use of learned models or neural networks $(N N)$. During this reduction process a lot of assumptions are made which possibly affect the usability of the reduced model e.g. elimination of high-frequency effects within the system behavior, linearization of the behavior within fixed valid ranges, etc. Whether or not the accuracy of the reduced model is affected by the reduction process depends heavily on the used reduction techniques. This reduced model can subsequently be used in the deployment process. Again, some assumptions are made during this process which possibly affect the usability and accuracy such as fixed-point transformations, discretization, etc. Problems may arise if these assumptions are not captured which negatively influence the model (re-)usability as the assumptions are necessary to assure correct use of the reduced models (Zeigler, B. P. 1984).

In our approach we try to overcome the aforementioned problems by explicitly defining the range-of-validity of each model-of-the-physics using some key elements of the frames concept (Denil et al. 2017). More specifically, we capture the essential meta-information for valid deployment of models-of-the-physics in a Validity Frame (VF). This meta-information can be used not only to assess the valid deployment of physicsbased models, it will also allow to assess the computation-memory-accuracy trade-off, which is deemed necessary for running physics-based models onto various constrained embedded controllers.

The rest of the paper is organised as follows : Section 2 presents some essential background and related work on frames. Section 3 defines a running example. Section 4 discusses the basic concepts of the presented solution. Section 5 implements a case study and finally, Section 6 concludes the paper and defines the future work.

\section{RELATED WORK}

The frames concept is not a novelty but has been around since the early 1980's when Zeigler (Zeigler, B. P. 1984) defined the original "experimental frames" idea. These experimental frames (EF) helped documenting the meta-information necessary to execute the model itself. The focus of his work was primarily on the activity of model execution and simulation. Extending the work of Zeigler in developing a theory and framework for modelling and simulation, Traore and Muzy (Traore and Muzy 2006) formalized experimental frames to describe the experimental contexts in which a simulation model is a valid representation of a system and consequentially describe the valid range of the model itself. More recent work (Schmidt, Durak, and Pawletta 2016) show how to use experimental frames for model-based testing of simulation models.

(Denil et al. 2017) observed that a model's frame depends on the activity that is performed and describes why different activities require different frames. They proposed the validity frame concept which defines the experimental context of a model in which that model gives predictable results. However, they do not provide a meta-model of the concept. In this work, this validity frame idea will be used and extended.

\section{RUNNING EXAMPLE}

We use a running example to clearly show the need for the validity frame concept and to point out what meta-information is important for valid deployment of models-of-the-physics. The example is a case study of a cyber-physical problem where a small car launches a ball in the air and tries to catch it before it hits the 
ground, as shown in Figure 1. This straightforward example is ideal as it is easy to understand but complex enough, as numerous physical assumptions are made during the development of the physical models.
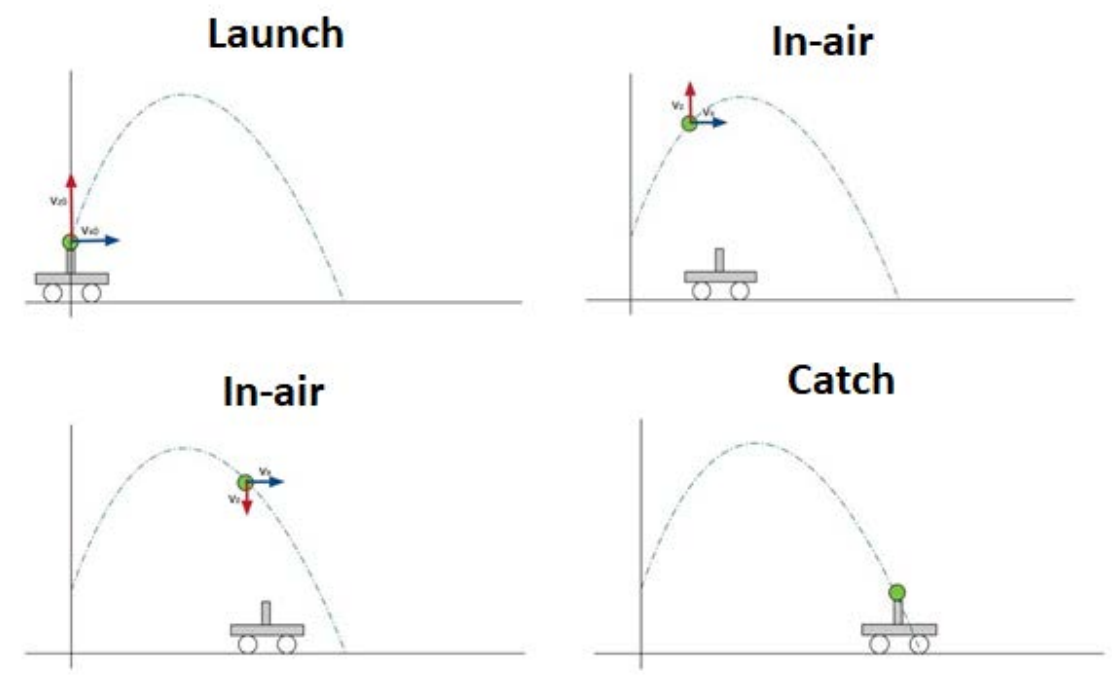

Figure 1: Flying ball use case.

In the flying ball use case, two different physical bodies are interacting, namely (i) the ball launched in the air and (ii) the car driving on the road. We only consider the model-of-the-physics of the ball. The physical behavior of the car is out of scope for this running example. The following physics-based models for the ball were already developed for other purposes in a previous case study:

- High-fidelity model: This model is the most detailed and accurate model developed using the Siemens system simulation tool Simcenter Amesim (Simcenter Amesim 2018). It was used as back-to-back(b2b) test reference model to analyze the accuracy of the derived reduction models.

- Mathematical reduction: First reduction technique used was deriving the physical equations of the flying ball. In this mathematical model, we assume that the magnus effect of the ball (Briggs 1959) could be ignored and that the mass of the ball was small enough to generalize the gravitational forces on the ball. The air resistance of the ball is also constant and we consider the ball only flying in the lowest atmospheric layer.

- Learned model: For this learned model, the high-fidelity model was used as input. The assumptions made for the mathematical model are also valid for the learned model but additionally, the learned model restricts the weight of the ball to a constant value.

- Property-reduced model: For the property-reduced model, the amount of analyzable properties was reduced, more specifically the Z-component (height) of the flying ball was eliminated as this was not required for the usage in the previous case study.

The already available models are shown in Figure 7, depicted as "Existing representations" (black circles), together with the corresponding accuracy, memory and computation estimations. These parameters are very important for valid deployment of models-of-the-physics on constraint embedded systems and depend on different configurations. We will capture this as part of the range-of-validity in the validity frames concept, discussed in the subsequent section. 


\section{VALIDITY FRAME CONCEPT}

We define a component as main building block of a system. This is inspired by Component-Based Software Engineering approaches (CBSE) (Cai et al. 2000) as the primary objective of CBSE and the validity frame concept are aligned, namely increasing component (re-)usability. The general definition of the validity frame is given in (Denil et al. 2017) as the experimental context of a model in which that model gives predictable results. More specifically, the validity frame will be used to (i) capture the range-of-validity of a model and (ii) define methods/processes to assure that a model faithfully represents the original system. We conceptually divided the validity frame in 3 main parts:

- Meta-data: Defines the meta-information usable as input for the design space exploration (DSE) or search algorithms.

- Operational: Defines the model scope and usage context.

- Process: Defines the workflows to assess the model validity.

In Figure 2, the core part of the meta-model of the validity frame concept is shown, specific for valid deployment of simulation models on constrained embedded systems. Some similarities exist between this meta-model and the one of a Functional Mock-up Unit(FMU), defined in (FMI std. 2018). Note that, for simplicity, we do not give the complete meta-model e.g. the relations between the experiment processes are not shown.

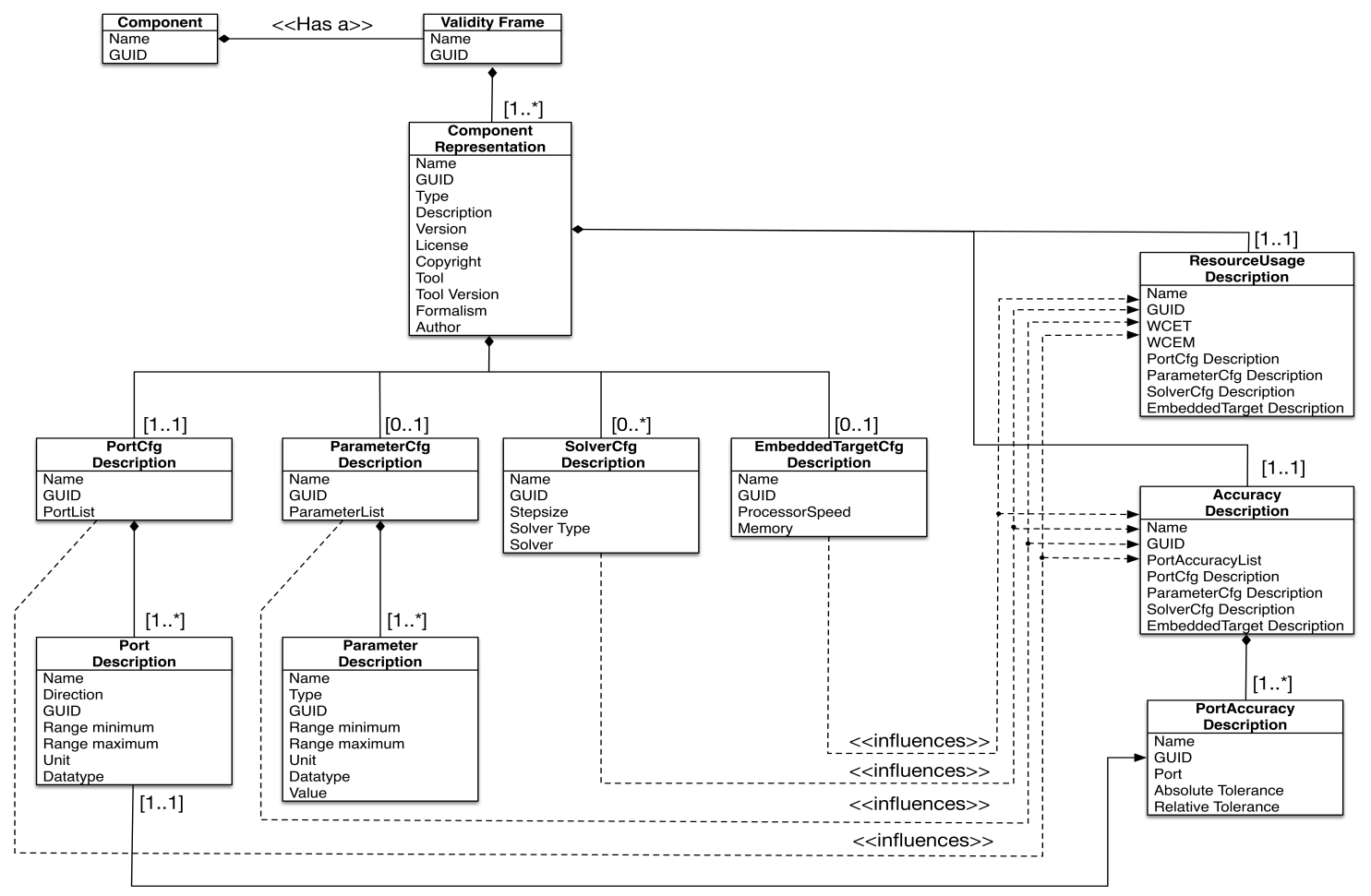

Figure 2: Validity frame meta-model.

In our validity frame concept, we do not restrict ourselves to models to represent the component functional and/or non-functional behavior. We define the ComponentRepresentation or short representation as a faithful representation of the component or system. This representation can either be a model, source code or source code binary. A validity frame can contain multiple representations of one component as 
different variants of a ComponentRepresentation can exist, caused by different reductions, different representation scoping, different experimental environments, etc. A hierarchical structure emerges because all the contained representations are related, either as variants representing the same component or as derived representations. This is what we call a representation chain of a component, an example shown in Figure 7. This hierarchical representation chain enables continuous traceability to the component and related component representations, which eases the assessment of the range-of-validity of each representation.

\subsection{Meta-data and operational part}

An important part of the validity frame is the definition of the range-of-validity of the representation. This depends on numerous aspects of the representation and its execution environment. First aspect is the interface definition, captured in PortCfgDescription. As shown in the running example of Section 3, not all representations need to have the same interfaces (property-reduced model does not contain an interface for the z-component, unlike the other representations). The PortCfgDescription contains a list of ports defined by the following attributes:

- Direction: Direction of the port (input/output).

- Range minimum/maximum: The admissible signal segment for this interface (Zeigler, B. P. 1984).

- Unit: The unit of measurement of the interface signal.

- Datatype: The data type of the interface signal.

The next aspect is the parameter configuration captured in ParameterCfgDescription. Again as shown in the running example, not all representations need equal calibration possibilities (ball weight of the learned model cannot be calibrated, unlike the other representations). The ParameterCfgDescription contains a list of parameters, which are defined using the same attributes of the port object. The only difference is the value which indicates the selected value for that parameter. Note that the interface and parameter definition is closely related to what properties are taken into account when modeling the component. To enable property reasoning, the relation between the interfaces and parameters and their physical meaning needs to be standardized using a formally defined ontology (Vanherpen et al. 2016).

The last aspect that influences the representation validity is the execution environment. The representations of the component can either be used in a simulation environment or can be executed on an embedded target. The necessary meta-data to define these execution environments are correspondingly captured in SolverCfgDescription and EmbeddedTargetCfgDescription. The SolverCfgDescription is closely related to (Ewald and Uhrmacher 2014). In this work, the authors defined a domain-specific language for simulation experiments. Based on this, we specify the simulation environment or simulation experiment using the following essential attributes:

- Solver: The selected solver.

- Solver type: The type of solver used.

- Stepsize: The sample time used with the corresponding solver.

The EmbeddedTargetCfgDescription contains relevant meta-information about the embedded target on which the representation is running. This is defined using the following attributes:

- ProcessorSpeed: The maximum clock frequency of the processor.

- Memory: The maximum available memory of the embedded target. 
All the aforementioned aspects have influence on (i) the representation accuracy and (ii) the representation resource usage. The accuracy of a representation is defined as the accuracy of each output interface against the output interfaces of a reference component. This reference component, also known as golden reference, can either be the real component or a high-fidelity representation of the component. The AccuracyDescription contains a list of port accuracy descriptions defined by either the relative or absolute tolerance against the reference interface signal. To ensure reproducible accuracy analyzes, the accuracy analysis methods are captured within the VF. The ResourceUsageDescription contains the essential meta-information of the deployed representation resource usage on the defined embedded target using the following attributes:

- WCET: Worst-case execution time of the representation. This timing metric is measured on the real embedded target using a specialized timing analysis tool.

- WCEM: Worst-case memory consumption of the representation. This memory metric is an estimate calculated when generating the source-code for a specific embedded target.

Note that the memory and timing metrics need to be measured on the real embedded target using specialized analysis tools. Analyzing these metrics can be expensive for representations build from scratch but can be justified by the potential re-usability benefit.

\subsection{Process part}

Another key part of the validity frame are methods/processes to assure that a representation faithfully represents the component. This means we need to assess the accuracy of the representation against the component itself or against a selected representation of the component, also referred to as the golden reference. This is possible by performing a back-to-back (B2B) experiment. This B2B experiment plays a pivotal role in the re-usability of the component representations. It will allow to extend the usability of a representation by performing extra experiments, and by this assessing the accuracy and possibly resource usage, while altering the operational part of the representation. This B2B experiment is modeled within the validity frame to enable automatic and valid experimenting. Figure 3 shows the experimental setup for a B2B experiment,

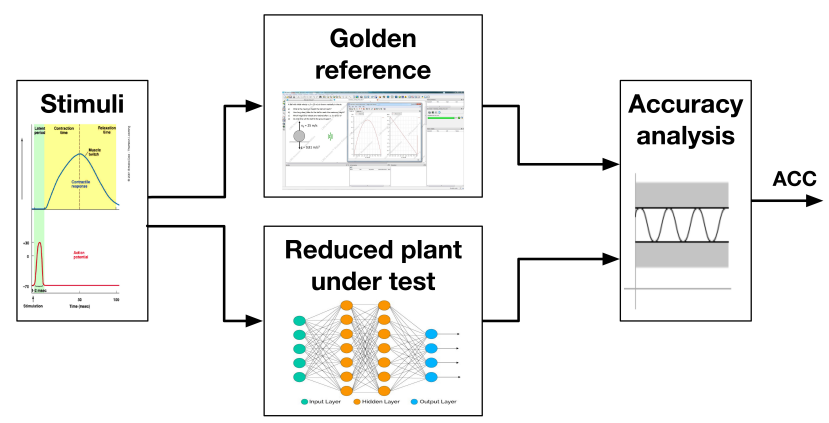

Figure 3: Experimental setup of back-to-back experiment.

used for the accuracy assessment. The key parts, modeled explicitly in the process part of the validity frame, are:

- Experiment harness: Defines the wiring of the experimental setup.

- Stimuli: Defines the input traces used to perform a valid B2B experiment.

- Golden reference: The reference component used as reference for the B2B experiments.

- Accuracy analyzer: The definition of how to assess the representation accuracy. 
We define a re-use process, based on the validity frame concept, that uses this explicitly modeled B2B experiment to increase representation (re-)usability. The re-use process is shown in Figure 4. It shows that we first explore the existing representations of a component. If none of the representations are sufficient for the system, we take the best-fit representation and tweak the operational part, trying to adapt it to the system requirements. After adapting the representation, we perform a B2B experiment to assess the accuracy and analyze the resource usage. If this fits all the system requirements, a new representation is added to the validity frame.

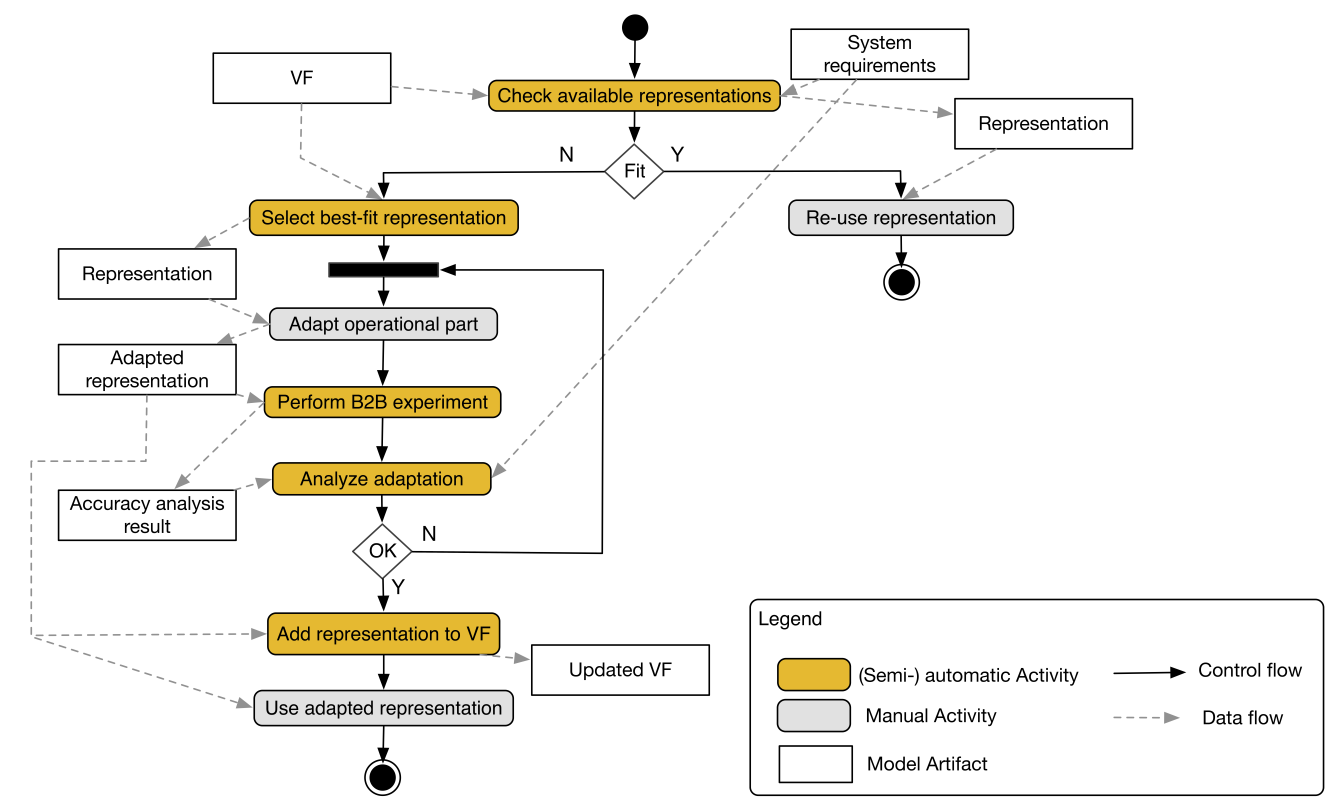

Figure 4: Re-use process with VF update.

\section{CASE STUDY}

In this section, we use a model predictive control (MPC) system as academic use case to illustrate the proposed concepts of the previous section. The MPC system will try to catch a launched ball by controlling the propulsion force of the car, as defined in Section 3. The MPC is a form of control in which the current control action is obtained by solving, at each sampling instant, a finite horizon open-loop optimal control problem using a physics-based model (D.Q.Mayne et al. 2000). In Figure 5, a general scheme of a MPC is given where the predicted output is used to predict and optimize the control input in order to adhere to a reference trajectory. Both the Prediction horizon $\mathrm{n}_{\text {prediction }}$ and Control horizon $\mathrm{n}_{\text {control }}$ can be adapted depending on the dynamics of the system under control. Specifically for this case study, the trajectory of

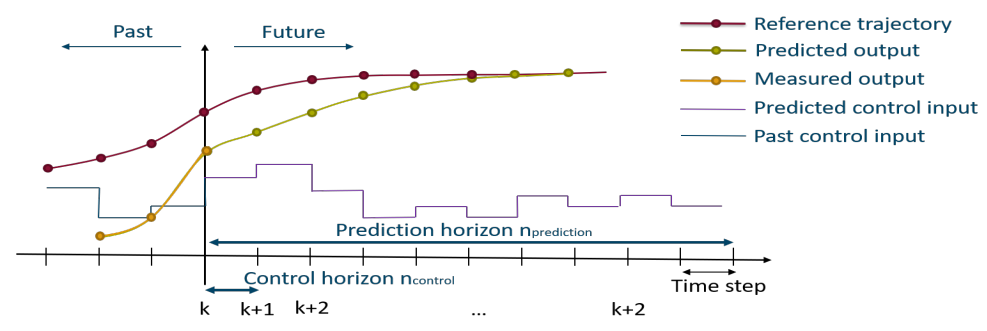

Figure 5: MPC past and future trajectories. 
the flying ball is predicted, using a physics-based model of the ball, over a finite prediction horizon. This prediction horizon is used to optimize online the optimal control problem where the propulsion force of the car is optimized in order to minimize the error between the x-position of the ball and the x-position of the car. The conceptual architecture of the MPC for this case study is shown in Figure 6.

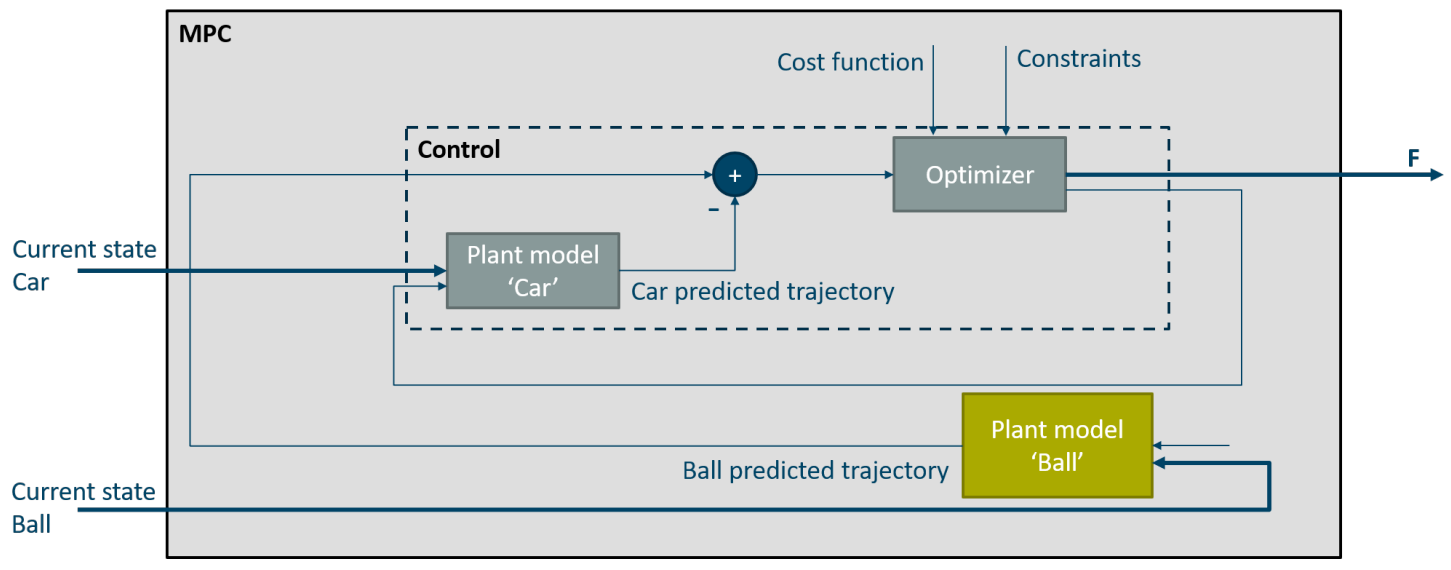

Figure 6: Model predictive control case study.

The correct behavior of the system, namely catching the flying ball, depends heavily on the validity range of the physics-based models for both the car and ball model. For simplicity, we focus only on the ball model rather then on both physics-based models. This means that the control component contains both the optimizer function and the physics-based model of the car. The following requirements are defined for the integration of the MPC controller:

- Control component - ball model interface is the predicted x-position of the ball $\mathbf{x}_{\mathbf{b a l l}}[\mathrm{cm}]$.

- The weight of the ball $\mathbf{m}_{\text {ball }}=0.50[\mathrm{~kg}]$.

- The system operates indoor (no turbulent air, gravitational acceleration $\mathbf{g}=9.81 \mathrm{~m} / \mathrm{s}^{2}$ ).

- The desired accuracy of the predicted x-position of the ball $\mathbf{A} \mathbf{C C}_{\text {desired }}=75$ [\%] (experimental setup definition as defined in the process part: relative to high-fidelity model - ODE4 fixed-step solver - stepsize 0.01s).

- Output of the MPC controller is propulsion force $\mathbf{F}[\mathrm{N}]$.

- $\quad$ Prediction horizon $\mathbf{n}_{\text {prediction }}=10$.

- Control horizon $\mathbf{n}_{\text {control }}=1$.

- $\quad$ Bounded optimization loops $\mathbf{n}_{\text {optimization }}=50$.

- Control loop period $\mathbf{T}=100 \mathrm{~ms}$.

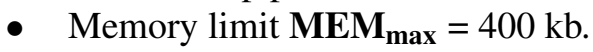

These system requirements need to be taken into account when integrating the control component and the physics-based ball component. After defining the integration model, which is a causal block diagram, all system requirements can be automatically checked using the meta-information defined in the validity frame of both components. We will try to re-use the already defined physics-based representations of the running example of Section 3 using the re-use process defined in Figure 4. This way, we can use the range-of-validity, defined in the validity frames, to select an optimal, scenario adapted, representation of the ball model. 


\subsection{Design space exploration of re-use components}

First step in the re-use process using validity frames is checking if a well-suited representation of the ball model exists which adheres to the aforementioned system requirements. This exploration phase is divided in the following subsequent analysis steps.

\subsubsection{Integration analysis}

First step is performing static checks on the controller integration model, namely checking the interconnected interfaces and parameters compatibility of the physics-based model. Some simple integration checks are automatically performed using the meta-information defined in the validity frames e.g. unit check of signal, data-type check of signal, parameter range check etc.

In the case study, the integration model is valid for all the representations of the flying ball model. Some representations have the z-position of the flying ball, which is not really required but these representations are still valid within the current scenario.

\subsubsection{Accuracy analysis}

Next step is performing the accuracy analysis where we check if the desired accuracy is met with the specified conditions. In this case study, the desired accuracy is specified for the x-position of the ball model in a simulation environment with the fixed-step Runge-Kutta ODE4 solver and a stepsize of 0.01 seconds, as modeled in the back-to-back experiment in the process part of the VF. In order to analyze the accuracy for the ball model representations, some experiments need to be executed as not all the representations are tested using the exact same solver, which can result in different precision. These experiments are automatically triggered and the back-to-back experiment, as shown in Figure 3, is generated and automatically executed. After executing this experiment and acceptance of the adaptations, the validity frame of the ball model is updated. More specific, a new representation is added to the validity frame with the new range-of-validity description and updated accuracy number. The updated validity frame representation chain with accuracy numbers is shown in Figure 7. Two representations were added to the already existing representation chain, namely the second mathematical reduction and second property reduction.

\subsubsection{Memory analysis}

Next step is checking the embedded target memory constraints. For each of the integrated components, a worst case memory estimation is defined in the validity frame. In order to check the memory constraints, we calculate the memory usage of the integration model by adding the individual memory estimates of each integrated component. The use case specific equations are:

$$
\begin{gathered}
M E M_{\text {control }}=M E M_{\text {car }}+M E M_{\text {optimizer }}=340 \mathrm{~kb}, \\
M E M_{\text {total }}=M E M_{\text {control }}+M E M_{\text {ball }} .
\end{gathered}
$$

In the case study, we assume that the memory consumption of the control component is fixed and cannot be altered. This way, we can easily derive the maximum possible memory consumption for the physics-based model of the ball, namely $60 \mathrm{~kb}$. By looking to the representation chain in Figure 7, we notice that all representations have a smaller memory footprint, except for the learned model. We manually reduced this learned model to meet the memory constraints and checked the accuracy via a back-to-back experiment and updated the validity frame by adding the second learned model representation. 


\subsubsection{WCET analysis}

Last step is checking the embedded target computational constraints. For each of the integrated components, a worst case execution time (WCET) estimation is defined in the validity frame. In order to check the computational constraints, we calculate the WCET of the integration model by adding the individual WCETs of each integrated component. Note that the number of iterations at which a component is executed per time step needs to be taken into account. The use case specific equations are:

$$
\begin{gathered}
W C E T_{\text {control }}=W C E T_{\text {car }} * n_{\text {prediction }} * n_{\text {control }}+W C E T_{\text {optimilization }} * n_{\text {optimalization }} * n_{\text {control }}, \\
W C E T_{\text {total }}=W C E T_{\text {control }}+W C E T_{\text {ball }} * n_{\text {prediction }} * n_{\text {control }} .
\end{gathered}
$$

In the case study, we assume that the WCET of the control component is fixed and cannot be altered. This way, we can easily derive the maximum WCET for the physics-based model of the ball, namely $3 \mathrm{~ms}$. In Figure 7, we notice that only the second learned model meets the WCET constraint. We manually reduce the mathematical model and verified the accuracy against the high-fidelity model and again updated the validity frame. We did not reduce the second property-reduction model as the accuracy was already less than other representations.

\subsection{Representation selection}

In the case study, we started with 4 representations, 1 high-fidelity model and 3 types of reduced models for the physics-based model of the ball. During the re-use process, we extended the validity frame of the ball with 4 extra representations in order to find a suited representation that meets all system requirements. In Figure 7, the complete representation chain of the ball model validity frame is shown. From this graph, we can see that the second learned model is the best-fit representation and this representation is selected to integrate in the MPC controller. Note that the second mathematical model is also a valid candidate but this representation is less accurate than the selected representation.

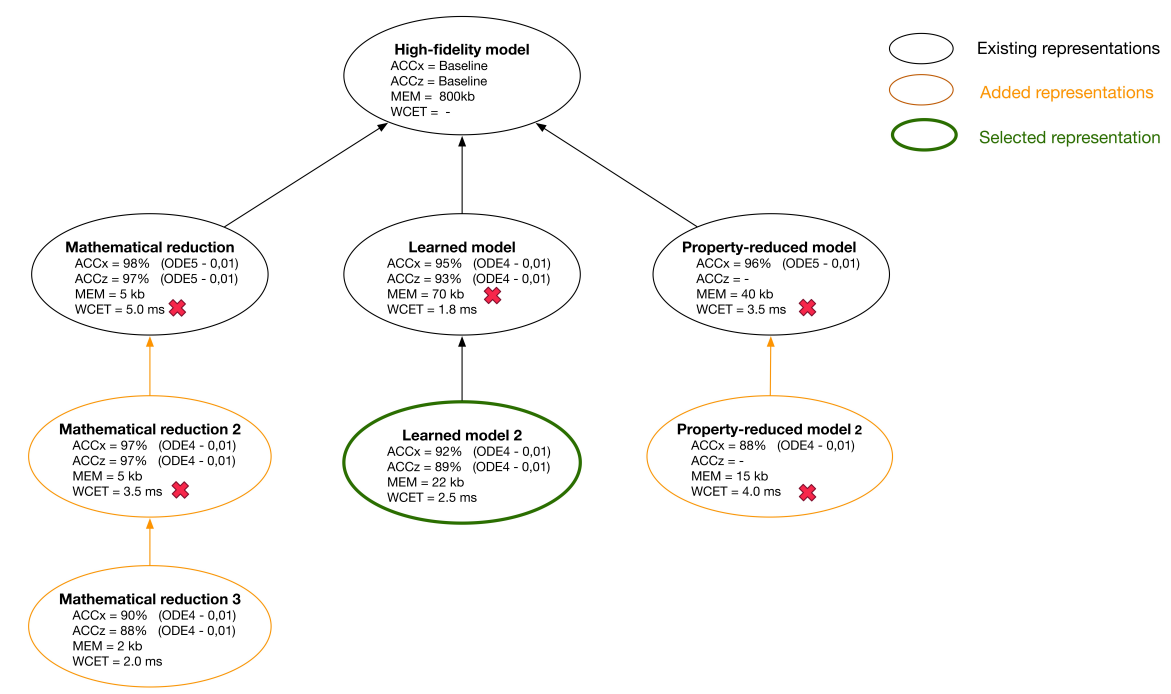

Figure 7: Validity frame representation chain - case study specific. 


\section{DISCUSSION AND FUTURE WORK}

This paper presented a framework to ease the valid deployment of models-of-the-physics on constrained embedded targets and to increase the re-usability of these models. This is achieved by explicitly capturing the range-of-validity within a validity frame and providing a (semi-)automated experimenting process. A re-use process is defined based on the presented validity frame concept and experimenting process.

In the future, we plan to extend the proposed methodology by increasing the captured meta-information with the focus on the usage in advanced control such as virtual sensors, adaptive control systems, etc. A startingpoint for this extension will be the OMG MARTE Profile (MG 2009), as they already define various standard constructs to express non-functional properties, time-related constraints and a way to describe the execution platform (hardware and software). We will also extend the process part by providing more automated processes such as calibration and testing processes. This will increase the validity frame usage, and by this increase model re-usability, throughout the complete development cycle. Next, we aim to facilitate the usage of validity frames by providing one or more domain-specific languages and tool-support to specify, verify and validate the range-of-validity. We will also investigate how to manage the consistency within the representation chains and the related validity frames.

\section{ACKNOWLEDGMENT}

This work has been carried out within the framework of ITEA 3, EUREKA Cluster programme project 'EMPHYSIS' (Embedded systems with physical models in the production code software, HBC.2017.0290) funded by the agency Flanders Innovation \& Entrepreneurship (VLAIO).

\section{REFERENCES}

Briggs, L. J. 1959. "Effect of Spin and Speed on the Lateral Deflection (Curve) of a Baseball; and the Magnus Effect for Smooth Spheres". American Journal of Physics vol. 27 (8), pp. 589-596.

Cai, X., M. R. Lyu, K.-F. Wong, and R. Ko. 2000, Dec. "Component-based software engineering: technologies, development frameworks, and quality assurance schemes". In Proceedings Seventh Asia-Pacific Software Engeering Conference. APSEC 2000, pp. 372-379.

Denil, J., S. Klikovits, P. J. Mosterman, A. Vallecillo, and H. Vangheluwe. 2017. "The Experiment Model and Validity Frame in M\&\#38;S”. In Proceedings of the Symposium on Theory of Modeling \& Simulation, TMS/DEVS '17, pp. 10:1-10:12. San Diego, CA, USA, Society for Computer Simulation International.

D.Q.Mayne, J.B.Rawlings, C. Rao, and P. Scokaert. 2000. "Constrained model predictive control: Stability and optimality". Automatica vol. Volume 36, Issue 6, pp. Pages 789-814.

EMPHYSIS 2018. "EMPHYSIS - Embedded systems with physical models in the production code software”. https://itea3.org/project/emphysis.html. Sep 2017 - Aug 2020.

Ewald, R., and A. M. Uhrmacher. 2014, February. "SESSL: A Domain-specific Language for Simulation Experiments". ACM Trans. Model. Comput. Simul. vol. 24 (2), pp. 11:1-11:25.

FMI std. 2018. "FMI - Functional Mock-up Interface”. https://fmi-standard.org.

Lee, E. A. 2008, 05. “Cyber Physical Systems: Design Challenges”. In 2008 11th IEEE International Symposium on Object and Component-Oriented Real-Time Distributed Computing (ISORC), Volume 00, pp. 363-369.

MG 2009. UML Profile for MARTE: Modeling and Analysis of Real-Time Embedded Systems. 
Pastorino, R., F. Cosco, F. Naets, W. Desmet, and J. Cuadrado. 2016, May. "Hard real-time multibody simulations using ARM-based embedded systems". Multibody System Dynamics vol. 37 (1), pp. 127143.

Schmidt, A., U. Durak, and T. Pawletta. 2016. "Model-based testing methodology using system entity structures for MATLAB/Simulink models". SIMULATION vol. 92 (8), pp. 729-746.

Simcenter Amesim 2018. "Siemens system simulation tool Simcenter Amesim". https://www.plm. automation.siemens.com/global/en/products/simcenter/simcenter-amesim.html.

Sánchez, L., I. Couso, J. Otero, Y. Echevarría, and D. Anseán. 2017. "A Model-Based Virtual Sensor for Condition Monitoring of Li-Ion Batteries in Cyber-Physical Vehicle Systems". Journal of Sensors vol. vol. 2017, pp. 12 pages.

Traore, M. K., and A. Muzy. 2006. "Capturing the dual relationship between simulation models and their context". Simulation Modelling Practice and Theory vol. Volume 14, Issue 2, pp. Pages 126-142.

Vanherpen, K., J. Denil, I. Dávid, P. D. Meulenaere, P. J. Mosterman, M. Torngren, A. Qamar, and H. Vangheluwe. 2016, April. "Ontological reasoning for consistency in the design of cyber-physical systems". In 2016 1st International Workshop on Cyber-Physical Production Systems (CPPS), pp. 1-8.

Zeigler, B. P. 1984. "Multifaceted Modelling and Discrete Event Simulation”. London, Academic Press.

Zeyu, C., X. Rui, W. Chun, and C. Jiayi. 2017. "An on-line predictive energy management strategy for plugin hybrid electric vehicles to counter the uncertain prediction of the driving cycle". Applied Energy vol. Volume 185, Part 2, pp. Pages 1663-1672.

\section{AUTHOR BIOGRAPHIES}

BERT VAN ACKER is a $\mathrm{PhD}$ student at the University of Antwerp, Belgium. He holds a master degree in Industrial Engineering Technology - Automotive Engineering at the University of Antwerp. His research interest includes Model-Based Engineering of complex cyber-physical systems. bert.vanacker@uantwerpen.be.

YURI DURODIE is a researcher at Siemens Industry Softwares in Leuven, Belgium. He holds a master degree in Electro-mechanical Engineering from the Free University of Brussels (VUB). His research interest includes advanced controls in automation and robotisation. yuri.durodie@siemens.com.

ALEXANDER VAN BELLINGHEN is a research engineer at Siemens Industry Software in Leuven, Belgium. He obtained his master's degree in electro-mechanical engineering at KU Leuven and focusses on embedded controls. alexander.van_bellinghen@siemens.com.

KRIS VANSTECHELMAN leads an Embedded Software and Hardware Engineering team at Dana Belgium, Bruges. He holds a master degree in Electromechanical Engineering from KU Leuven Faculty of Engineering Technology, Ghent Technology Campus. His main research area of interest is developing new methodologies, processes \& supporting tools for design \& testing of safety critical embedded systems for powertrain applications. kris.vanstechelman@dana.com.

PAUL DE MEULENAERE holds a $\mathrm{PhD}$ in physics and is professor at the University of Antwerp, faculty of Applied Engineering. He is also associated to the Flanders Make research centre. His research is focused on the design of embedded technology for cyber-physical systems. paul.demeulenaere@uantwerpen.be.

JOACHIM DENIL is currently an assistant professor at the university of Antwerp. He is also the Univ. of Antwerp core-lab manager in Flanders Make. Joachim's research combines aspects from computer science, electronics and simulation to support engineers in the model-based design of cyber-physical systems. joachim.denil@uantwerpen.be. 